\title{
Curly: An AI-based Curling Robot Successfully Competing in the Olympic Discipline of Curling
}

\author{
Dong-Ok Won ${ }^{1}$, Byung-Do Kim ${ }^{1}$, Ho-Jung Kim ${ }^{1}$, Tae-San Eom ${ }^{1}$, \\ Klaus-Robert Müller ${ }^{1,2}$, and Seong-Whan Lee ${ }^{1}$ \\ ${ }^{1}$ Department of Brain and Cognitive Engineering, Korea University, Seoul, Republic of Korea \\ ${ }^{2}$ Department of Computer Science, Berlin Institute of Technology, Berlin, Germany \\ sw.lee@korea.ac.kr
}

\begin{abstract}
Most artificial intelligence (AI) based learning systems act in virtual or laboratory environments. Here we demonstrate an AI-based curling robot system named 'Curly' that competes on a real-world curling ice sheet. Curly encompasses (1) an AI-based curling strategy and simulation engine under consideration of the high 'icy' uncertainty, (2) the thrower robot enabled by autonomous driving with traction control, and (3) the skip robot that allows to recognize the curling field and stone configuration based on vision technology. The Curly performed well both: in classical game situations and when interacting with human opponents, namely, the top-ranked Korean amateur high school curling team.
\end{abstract}

\section{Introduction}

Numerous attempts have been made to apply AI technologies to real-world outside confined laboratory environments [Archibald et al., 2009; Yee et al., 2016; Ahmad et al., 2016]. This is a hard problem since the real-world has numerous variables that vary over time, which can have a profound effect on the performance of AI. Moreover, the real-world has high uncertainties, which are too complex and too ill-defined to be modeled with the necessary accuracy. Thus it becomes necessary to encompass uncertainty into the modeling and furthermore to measure and approximate changes in real-world environments.

This work has chosen to use the complex game of curling as a test bed for demonstrating the interaction of an AI system and the real(slippery) world.

Curling (an Olympic discipline) is a turn-based game in which two teams play alternately on the ice sheet, requiring a high level of strategic thinking and performance. When we look at curling from an AI perspective, comparing with board games such as chess or go [Silver et al., 2016; 2017], the following two parts are significantly different. Firstly, curling has a considerable

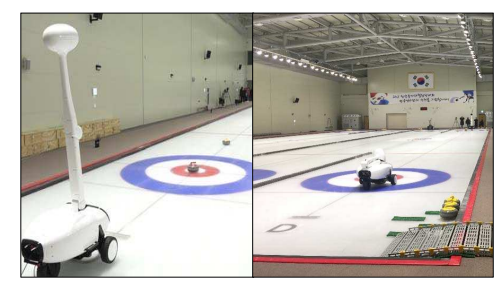

Figure 1: The AI curling robots play the curling game on a real curling ice sheet. (Left) The skip robot recognizes the game state using the location of stones. (Right) The thrower robot is throwing in order to deliver the stone to the target location chosen by the AI strategy engine. https://youtu.be/yXygf8oz58k

amount of legal moves because the game progresses in continuous space [Yee et al., 2016; Ahmad et al., 2016]. Secondly, because of stones' collisions, curling requires a sophisticated and time-consuming physics-based simulation process to accurately describe and simulate possible legal moves for the next step [Ito and Kitasei, 2015; Ohto and Tanaka, 2017]. In recent years, studies on strategy-AI in curling area have been actively conducted in a virtual simulator environment [Yee et al., 2016; Ahmad et al., 2016]. In reality, however, ice has strongly varying conditions etc. so the uncertainties are higher than they may be expected from a simulation in the virtual world.

Note that the real curling game is played on the ice sheet covered with pebbles. The pebbles change their condition from time to time depending on the temperature, humidity, ice makers, elapsed time since the maintenance ended, amount of sweeping done during the game, etc [Denny, 2002; Nyberg et al., 2013]. That is, when delivering curling stones using the same direction, force and curl, the trajectory of the stones will vary strongly over time. In addition, most of the strategic plays take place within the 1.83 meter radius of house region about 40 meters away. Compared with rather controlled (virtual) board games application (i.e., chess, go, etc.), the real-world application of curling AI is somewhat challenging. 


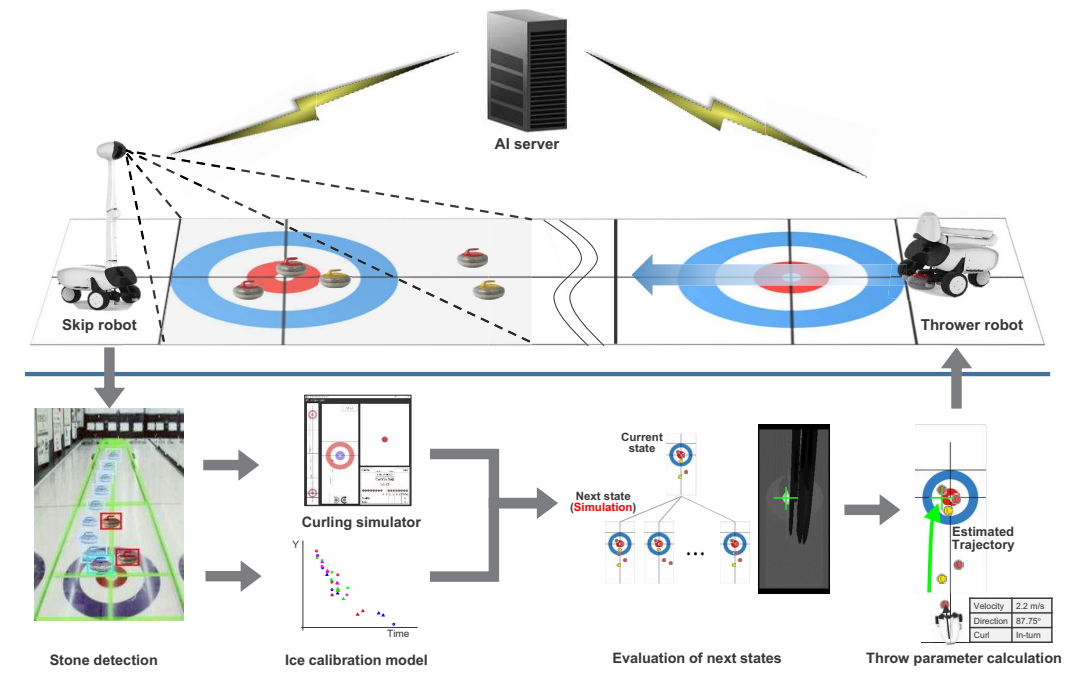

Figure 2: Proposed AI Curling Robot System: Curly. The system consists of thrower/skip robots, curling simulator, and strategyAI. The system operation process consists of 3 steps as follows. 1) The skip robot recognizes the coordinates of the stones on the ice sheet and transmits them to the main server. 2) After receiving the coordinates, the main server establishes an AI-based strategy taking the uncertainty into account and sends it to the thrower robot. 3) The thrower robot delivers a stone with the strategy received from the server, and when all the stones stop, the opponent takes his turn again.

\section{Methods}

\subsection{Curling Artificial Intelligence System}

The main server part of AI curling robot system has the strategy-AI and curling simulator (Figure 2). StrategyAI finds the optimal target position based on the stone's coordinates, the estimated uncertainty and the current game status. We developed a physics-based curling simulator which is designed to adjust the parameters (i.e., throw angle, velocity, and curl direction, etc.). This simulator can help our AI to establish an optimal strategy in the real ice sheet environment. Intelligent compensation for uncertainty allows the robot to throw stones precisely into the desired target points on the real ice sheet environment (Figure 2) although all individual sub-components of our AI system are limited by errors of uncertainty.

\section{Ice Calibration}

The most striking difference between curling and other games is that it is almost impossible for a human or robot thrower to send the stone to the desired location (note that human players may in addition be hampered in their precision by being nervous). Calibration is to match the difference of the trajectory between the real ice sheet environment and the virtual physics simulator environment. It should be noted that the established strategies are quite different according to how precisely the inevitable uncertainty is approximated even in the same game situation.

\section{Strategy-AI}

We developed a strategy-AI based on reinforcement learning and tree search algorithm using physics-based simulations and evaluation values. To increase the success rate of the strategy regardless of uncertainty, we consider the uncertainty area that represents possible reaching target points of thrown stone. In this manner, it becomes possible to perform not only stable but also competitive strategies.

\subsection{Curling Robots}

We constructed two identical robots (operated as skip and thrower modes respectively), each equipped with video analysis, data communication and throwing control modules including traction control. The thrower robot performs strategy on the ice sheet while holding and rotating a curling stone and releasing the stone by unfolding the gripper (Figure 1). The skip robot can recognize the coordinates of the stones or the trajectories of the moving stones (Figure 1).

\section{Discussion and Conclusion}

In the present study, an AI-based curling robot system that clearly shows the feasibility of applying AI technologies to highly uncertain real-world environments. Integrated planning, simulation, and uncertainty estimation were key to the successful and competitive throwing strategy in this icy environment.

Future studies will aim to use explainable AI techniques [Bach et al., 2015] to gain a better understanding of critical shot impacts, thus allowing Curly and its makers to learn better from their mistakes.

\section{Acknowledgments}

This work was supported by Institute for Information \& Communications Technology Promotion (IITP) grant funded by the Korea government (MSIT) (No. 2017-000521, 2017-0-01779). 


\section{References}

[Ahmad et al., 2016] Zaheen Farraz Ahmad, Robert C Holte, and Michael Bowling. Action Selection for Hammer Shots in Curling. In Proceedings of the Twenty-Fifth International Joint Conference on Artificial Intelligence (IJCAI), pages 561-567, 2016.

[Archibald et al., 2009] Christopher Archibald, Alon Altman, and Yoav Shoham. Analysis of a Winning Computational Billiards Player. In Proceedings of the Eighteenth International Joint Conference on Artificial Intelligence (IJCAI), pages 1377-1382, 2009.

[Bach et al., 2015] Sebastian Bach, Alexander Binder, Grégoire Montavon, Frederick Klauschen, KlausRobert Müller, and Wojciech Samek. On pixelwise explanations for non-linear classifier decisions by layer-wise relevance propagation. PLoS ONE, 10(7):e0130140, 2015.

[Denny, 2002] Mark Denny. Curling rock dynamics: Towards a realistic model. Canadian Journal of Physics, 80(9):1005-1014, 2002.

[Ito and Kitasei, 2015] Takeshi Ito and Yuuma Kitasei. Proposal and Implementation of "Digital Curling". In Proceedings of the IEEE Conference on Computational Intelligence and Games (CIG), pages 469-473, 2015.

[Nyberg et al., 2013] Harald Nyberg, Sara Alfredson, Sture Hogmark, and Staffan Jacobson. The asymmetrical friction mechanism that puts the curl in the curling stone. Wear, 301(1-2):583-589, 2013.

[Ohto and Tanaka, 2017] Katsuki Ohto and Tetsuro Tanaka. A Curling Agent Based on the Monte-Carlo Tree Search Considering the Similarity of the Best Action Among Similar States. In Proceedings of the Fifteenth International Conference on Advances in Computer Games, pages 151-164, 2017.

[Silver et al., 2016] David Silver, Aja Huang, Chris J Maddison, Arthur Guez, Laurent Sifre, George Van Den Driessche, Julian Schrittwieser, Ioannis Antonoglou, Veda Panneershelvam, Marc Lanctot, et al. Mastering the game of Go with deep neural networks and tree search. Nature, 529(7587):484-489, 2016.

[Silver et al., 2017] David Silver, Julian Schrittwieser, Karen Simonyan, Ioannis Antonoglou, Aja Huang, Arthur Guez, Thomas Hubert, Lucas Baker, Matthew Lai, Adrian Bolton, et al. Mastering the game of Go without human knowledge. Nature, 550(7676):354359, 2017.

[Yee et al., 2016] Timothy Yee, Viliam Lisỳ, and Michael H Bowling. Monte Carlo Tree Search in Continuous Action Spaces with Execution Uncertainty. In Proceedings of the Twenty-Fifth International Joint Conference on Artificial Intelligence (IJCAI), pages 690-697, 2016. 